\title{
Bioincompatible Impact of Different Peritoneal Dialysis Fluid Components and Therapeutic Interventions as Tested in a Rat Peritoneal Dialysis Model
}

\author{
Andrea W. D. Stavenuiter, ${ }^{1}$ Karima Farhat, ${ }^{2}$ Margot N. Schilte, ${ }^{1}$ \\ Piet M. ter Wee, ${ }^{2}$ and Robert H. J. Beelen ${ }^{1}$ \\ ${ }^{1}$ Department of Molecular Cell Biology and Immunology, H269, VU University Medical Center, Van der Boechorststraat 7, \\ 1081 BT Amsterdam, The Netherlands \\ ${ }^{2}$ Department of Nephrology, VU University Medical Center, 1081 BT Amsterdam, The Netherlands
}

Correspondence should be addressed to Andrea W. D. Stavenuiter, a.stavenuiter@vumc.nl

Received 24 March 2011; Accepted 1 June 2011

Academic Editor: Hulya Taskapan

Copyright (c) 2011 Andrea W. D. Stavenuiter et al. This is an open access article distributed under the Creative Commons Attribution License, which permits unrestricted use, distribution, and reproduction in any medium, provided the original work is properly cited.

\begin{abstract}
Peritoneal dialysis (PD) is associated with functional and structural changes of the peritoneal membrane. In this paper, we describe the impact of different factors contributing to peritoneal incompatibility of PD fluid installation including presence of a catheter, volume loading, and the PD fluid components itself. These factors initiate recruitment and activation of peritoneal immune cells such as macrophages and mast cells, as well as activation of peritoneal cells as mesothelial cells in situ. We provide an overview of PD-associated changes as seen in our rat PD-exposure model. Since these changes are partly reversible, we finally discuss therapeutic strategies in the rat PD model with possible consequences of long-term PD in the relevant human setting.
\end{abstract}

\section{Factors Contributing to Functional and Structural Changes in an Experimental Rat PD Model: Introduction}

Peritoneal dialysis (PD) is a therapy used to replace kidney function in end-stage renal disease patients. The therapy is based on the ability of the peritoneal membrane to function as a dialysing membrane, allowing exchange of solutes and waste products between the PD fluids (PDFs) and the circulation. Dialysis fluid is instilled in the peritoneal cavity via a permanent catheter. PDFs contain an osmotic agent, mostly glucose, which facilitates fluid movement from the bloodstream to the peritoneal cavity leading to removal of metabolic waste products and water. Continuous removal of waste products achieved using PDs, results in improved well-being of patients. In contrast, in haemodialysis, waste products accumulate between two dialysis treatments. Furthermore, patients on PD have increased mobility compared to haemodialysis since $\mathrm{PD}$ can be done at home, and moreover, $\mathrm{PD}$ is less expensive.

However, drawbacks of PD include the risk of peritonitis and peritoneal membrane damage upon exposure to PDF. The latter induces inflammation, angiogenesis, and fibrotic changes. In this paper, we describe the usefulness of a rat PD exposure model in defining the impact of PDF components in this process. Finally, we will discuss interventions in this model and the possible clinical implications for long-term PD.

\section{PD-Related Changes as Observed in a Rat PD-Exposure Model}

The efficacy of PD depends on the structural and functional integrity of the peritoneum, that is, the inner surface of the abdominal wall (parietal peritoneum), omentum, and mesentery (visceral peritoneum). The peritoneum consists 
TABLE 1: The degree of contribution by extrinsic (catheter, uremia) and intrinsic (fluid) factors on the peritoneum in rats.

\begin{tabular}{|c|c|c|c|c|c|}
\hline \multirow{2}{*}{ Peritoneal changes } & \multicolumn{2}{|c|}{ Extrinsic factors } & \multicolumn{3}{|c|}{ Intrinsic factors } \\
\hline & Catheter & Uraemia & (Lactate) Buffer & Buffer + Glucose & Buffer + Glucose + GDP \\
\hline Ultrafiltration failure & - & \pm & \pm & + & ++ \\
\hline Angiogenesis & \pm & \pm & \pm & + & ++ \\
\hline Fibrosis & - & - & - & + & ++ \\
\hline Mesothelial regeneration & - & - & + & ++ & ++ \\
\hline Effluent cell number & - & - & - & + & + \\
\hline Omental mast cells & - & \pm & + & + & ++ \\
\hline Omental milky spots & - & - & + & + & ++ \\
\hline
\end{tabular}

GDP = glucose degradation products.

Peritoneal changes are scored from no $(-)$, weak $( \pm)$, and moderate $(+)$ to very strong alterations $(++)$ compared to control rats.

of a mesothelial cell (MC) monolayer and underlying connective tissue interstitium comprising extracellular matrix (ECM), blood vessels, fibroblasts, and innate immune cells.

In our rat PD-exposure model, we use normal renal function, no omentomectomy, and no addition of heparins during PD-exposure or addition of antibiotics [1]. We have shown that several weeks of PD results in a loss of MCs and denuded areas in the mesothelial layer [2]. Liver imprints of rats show increased mesothelial cell density, indicating mesothelial regeneration $[3,4]$. Among the mesothelial cells, vimentin-positive, spindle-like-shaped cells are found indicating the process of epithelial to mesenchymal transition (EMT; [5]). Changes also take place in the submesothelial interstitium, and ECM thickness is significantly increased upon PD [6-8].

Significantly higher numbers of leukocytes are found in the effluents of PD-treated animals compared to nontreated animals. Although the percentage of macrophages and lymphocytes does not change, an exchange of mast cells and eosinophils for neutrophils is seen after PD treatment $[7,9]$. Increased numbers of activated macrophages are seen in mesentery and omentum upon PD [5], including accumulations around vessel networks in the omentum, known as milky spots $[7,10]$. In a steady state, milky spots occupy a small percentage of the total surface area of the omentum, whereas after PDF exposure, this increases dramatically [3, 11]. Throughout the peritoneal tissues, new blood vessels and lymphatics [7] are formed. Angiogenesis leads to a large effective surface area exchange, contributing to ultrafiltration loss [12]. Furthermore, the thickened submesothelial fibrotic layer counteracts osmotic pressure further, reducing efficacy of exchange [13].

In summary, loss of mesothelium and induction of inflammation and angiogenesis are typical morphologic features seen in long-term PD which in part contribute to fibrotic changes, ultimately leading to technique failure.

In this paper, we discuss the changes that occur upon PD in our rat PD-exposure model. This model, as described above, shows similar changes upon long-term exposure to PDF fluid as patients on CAPD. For example, thickening of the submesothelial compact zone, degeneration of the MC layer, loss of microvilli on residual MC, and increased vessel numbers are also observed in biopsies from patients on long-term $\mathrm{PD}$, as in detail described by Williams et al. [14]. In addition, Devuyst et al. recently reviewed the similarities between mice, rats, and humans regarding a.o. peritoneal transport, aquaporins, and net UF after long-term PD exposure [15].

\section{Causes of PD-Induced Peritoneal Membrane Changes}

Several factors can contribute to PD-related changes, including the presence of the catheter, uraemia, peritonitis, and instillation of the volume loading of PDF itself. Moreover, different components of the PDF, including the used buffer (low $\mathrm{pH}$ ), glucose concentration, and glucose degradation products (GDPs) generated during heat sterilisation, influence peritoneal inflammation (as reviewed by Schilte et al. [2] and summarized in Table 1). The presence of only the catheter itself already induces PD-related morphological changes such as increase in angiogenesis and thickness of ECM, as well as slight effect on total effluent cell numbers $[9,16]$. Morphologic and inflammatory parameters of rats treated with PDF via a peritoneal catheter are significantly increased compared to rats treated via intraperitoneal needle injections (blood vessels, ECM thickness, and total cell count).

Volume loading is already a second inflammatory trigger. Zareie et al. showed that instillation of lactate buffer without glucose or GDPs already resulted in increased cell influx, mesothelial regeneration, angiogenesis, and increased number of milky spots although it not significantly enhanced fibrosis $[7,9]$. Moreover, they showed that the addition of glucose to the buffer-induced angiogenesis and mesothelial regeneration, and induced fibrosis as well as cell influx even further. Finally, the presence of GDPs enhanced all mentioned peritoneal changes, apart from cell influx and mesothelial regeneration, even further. Besides, GDPs, advanced glycation end products (AGEs) formed by heating of glucose, contribute to the toxicity of PDF [17].

It has become increasingly clear that more biocompatible PDFs (bicarbonate/lactate buffer, reduced concentration of glucose and GDPs) induce less damage and decrease impaired ultrafiltration when compared with conventional $\operatorname{PDF}[2,6,8,17]$. 
In addition, supplementing PDF with aminoguanidine, which scavenges GDPs and prevents AGE formation, resulted in less mesothelial denudation [18], reduced fibrosis, and less angiogenesis in omentum and parietal peritoneum, but not in mesentery [19] as shown in our own rat PD-exposure model.

Apart from intrinsic factors as PDF composition, other factors have been suggested to contribute to PD-related tissue remodelling (Table 1). Animal studies have shown the increase in vascular network as a result of uraemia in nonPD-treated animals. Although differences between uremic and nonuremic rats were found in control animals (no PD), there is no longer a prominent effect of uraemia during $\mathrm{PD}$ therapy $[4,20]$.

In contrast, peritonitis episodes always significantly contribute to peritoneal changes by inducing mesothelial damage, a massive inflammatory response, and increased vascularisation of peritoneal tissue leading to impaired membrane function $[12,21,22]$.

\section{Peritoneal Rest and Reversibility}

Activation of peritoneal cells, mediators, and pathways, results in functional and structural changes of peritoneal membranes in long-term PD. Some of these changes can be reversed by peritoneal rest. The reversibility of both morphological and functional alterations in the peritoneal membrane by peritoneal rest has been shown in animal studies $[23,24]$.

Peritoneal rest of more than 4 weeks restored ultrafiltration capacity as well as peritoneal permeability for glucose and total protein $[23,24]$. Moreover, the thickness of the parietal peritoneum was reduced, and omental and mesenterial vessel density was restored [23, 24]. Furthermore, peritoneal rest reversed the increased mast cell density and milky spot response and recovered PD-induced mesothelial damage [24]. These data suggest that PD-induced changes in the peritoneal membrane are reversible after peritoneal rest, at least in the rat model. We therefore foresee future therapies focusing on the prevention and reversibility of these changes as shown below. Moreover, this may have major implications for therapeutic clinical interventions in preventing the PDinduced changes in long-term PD.

\section{Therapeutic Interventions in the Rat PD Model}

Recent reviews of our group have shown that the rat PD model is also ideally to test anti-inflammatory therapeutic interventions $[2,25]$.

Heparin has been mentioned as a regulator in inflammation, but the addition of unfractionated or low-molecular weight heparin was not able to counteract the PD-fluidinduced changes in our rat PD model [5], but in an editorial, the usefulness was highlighted [26].

Recently, we could show that Sunitinib inhibits angiogenesis in our rat PD model, and so the usefulness of inhibitors in the field of angiogenesis can have important functional implications [27]. Moreover, the usefulness of Celecoxib as inhibitor of Cox-2 and the inflammation cascade has been discussed [28] and indeed in our rat PD model, we could demonstrate an effect on angiogenesis and functional ultrafiltration, but not on clear inflammation markers [29]. These two interventions so in part show some positive results, but future experiments should determine if no side effects of Celecoxib are seen, when used in a clinical setting (as presently is investigated in our institute) and whether the reduction of angiogenesis by Sunitinib really results in better ultrafiltration.

It must be mentioned that macrophages are thought to play a key role in the inflammatory process, as earlier discussed [2]. More recently, it was shown that the discrete balance between the so-called proinflammatory M1 macrophages and alternatively activated M2 macrophages might determine the outcome in inflammation in PD [30] as has also been shown for macrophages in wound healing [31] and oncology [32, 33].

Finally, molecules as bone morphogenetic protein-7 (BMP-7) and the vitamin D analogue paricalcitol have been shown to be important in inhibiting peritoneal and renal fibrosis [34, 35]. BMP-7 also was shown to prevent peritoneal damage in our PD-exposure model [36], and preliminary data on paricalcitol also show a clear effect in our PD rat model [37]. These observations should be investigated more deeply and mechanistically in the in vivo animal model, since they offer opportunities for therapeutic clinical interventions in the near future.

\section{Summary and Conclusion}

In long-term $\mathrm{PD}$, the catheter, uraemia, peritonitis, and permanent exposure to PDF (volume loading, glucose, and GDP's) result in morphological and functional changes of the peritoneal membranes. In part, the ultrafiltration loss seen in PD patients is caused by the bioincompatibility of PDF. Novel PDFs offer an improvement in biocompatibility, and further development of biocompatible fluids will lead to better preservation of the peritoneal membrane. Especially therapeutic interventions on angiogenesis, fibrosis, and inflammation are thought to be promising strategies in preventing ultrafiltration failure. We therefore foresee a combination therapy using more biocompatible fluids along with specific inhibitors involved in peritoneal inflammation/angiogenesis/fibrosis to be the most effective approach in future PD to prevent PD-induced changes and which will be beneficial for PD patients.

\section{Acknowledgment}

This work was supported by Grant no. C09.2331 of the Dutch Kidney Foundation.

\section{References}

[1] R. H. J. Beelen, L. H. P. Hekking, M. Zareie, and J. van den Born, "Rat models in peritoneal dialysis," Nephrology Dialysis Transplantation, vol. 16, no. 3, pp. 672-674, 2001. 
[2] M. N. Schilte, J. W. Celie, P. M. ter Wee, R. H. J. Beelen, and J. van den Born, "Factors contributing to peritoneal tissue remodeling in peritoneal dialysis," Peritoneal Dialysis International, vol. 29, no. 6, pp. 605-617, 2009.

[3] L. H. P. Hekking, M. Zareie, B. A. Driesprong et al., "Better preservation of peritoneal morphologic features and defense in rats after long-term exposure to a bicarbonate/lactatebuffered solution," Journal of the American Society of Nephrology, vol. 12, no. 12, pp. 2775-2786, 2001.

[4] M. Zareie, A. S. De Vriese, L. H. P. Hekking et al., "Immunopathological changes in a uraemic rat model for peritoneal dialysis," Nephrology Dialysis Transplantation, vol. 20, no. 7, pp. 1350-1361, 2005.

[5] M. N. Schilte, J. Loureiro, E. D. Keuming et al., "Longterm intervention with heparins in a rat model of peritoneal dialysis," Peritoneal Dialysis International, vol. 29, no. 1, pp. 26-35, 2009.

[6] S. Mortier, D. Faict, N. H. Lameire, and A. S. De Vriese, "Benefits of switching from a conventional to a low-GDP bicarbonate/lactate- buffered dialysis solution in a rat model," Kidney International, vol. 67, no. 4, pp. 1559-1565, 2005.

[7] M. Zareie, L. H. P. Hekking, A. G. A. Welten et al., "Contribution of lactate buffer, glucose and glucose degradation products to peritoneal injury in vivo," Nephrology Dialysis Transplantation, vol. 18, no. 12, pp. 2629-2637, 2003.

[8] M. Zareie, E. D. Keuning, P. M. ter Wee, R. H. Beelen, and J. van den Born, "Improvement of a chronic rat model for peritoneal dialysis by using heparin-coated catheters," Advances in Peritoneal Dialysis, vol. 20, pp. 150-154, 2004.

[9] M. Zareie, E. D. Keuning, P. M. ter Wee, C. G. Schalkwijk, R. H. J. Beelen, and J. van den Born, "Improved biocompatibility of bicarbonate/lactate-buffered PDF is not related to $\mathrm{pH}$," Nephrology Dialysis Transplantation, vol. 21, no. 1, pp. 208 216, 2006.

[10] N. Di Paolo, G. Sacchi, G. Garosi et al., "Omental milky spots and peritoneal dialysis-review and personal experience," Peritoneal Dialysis International, vol. 25, no. 1, pp. 48-57, 2005.

[11] R. H. J. Beelen, S. J. Oosterling, M. van Egmond, J. van den Born, and M. Zareie, "Omental milky spots in peritoneal pathophysiology (spots before your eyes)," Peritoneal Dialysis International, vol. 25, no. 1, pp. 30-32, 2005.

[12] K. N. Lai, S. C. W. Tang, and J. C. K. Leung, "Mediators of inflammation and fibrosis," Peritoneal Dialysis International, vol. 27, supplement 2, pp. S65-S71, 2007.

[13] A. Fusshoeller, "Histomorphological and functional changes of the peritoneal membrane during long-term peritoneal dialysis," Pediatric Nephrology, vol. 23, no. 1, pp. 19-25, 2008.

[14] J. D. Williams, K. J. Craig, N. Topley, and G. T. Williams, "Peritoneal dialysis: changes to the structure of the peritoneal membrane and potential for biocompatible solutions," Kidney International, vol. 63, no. 84, pp. S158-S161, 2003.

[15] O. Devuyst, P. J. Margetts, and N. Topley, "The pathophysiology of the peritoneal membrane," Journal of the American Society of Nephrology, vol. 21, no. 7, pp. 1077-1085, 2010.

[16] M. F. Flessner, K. Credit, K. Henderson et al., "Peritoneal changes after exposure to sterile solutions by catheter," Journal of the American Society of Nephrology, vol. 18, no. 8, pp. 22942302, 2007.

[17] K. Honda, K. Nitta, S. Horita et al., "Accumulation of advanced glycation end products in the peritoneal vasculature of continuous ambulatory peritoneal dialysis patients with low ultra-filtration," Nephrology Dialysis Transplantation, vol. 14, no. 6, pp. 1541-1549, 1999.
[18] E. A. Lee, J. H. Oh, H. A. Lee et al., "Structural and functional alterations of the peritoneum after prolonged exposure to dialysis solutions: role of aminoguanidine," Peritoneal Dialysis International, vol. 21, no. 3, pp. 245-253, 2001.

[19] M. Zareie, G. J. Tangelder, P. M. ter Wee et al., "Beneficial effects of aminoguanidine on peritoneal microcirculation and tissue remodelling in a rat model of PD," Nephrology Dialysis Transplantation, vol. 20, no. 12, pp. 2783-2792, 2005.

[20] S. Combet, M. L. Ferrier, M. Van Landschoot et al., "Chronic uremia induces permeability changes, increased nitric oxide synthase expression, and structural modifications in the peritoneum," Journal of the American Society of Nephrology, vol. 12, no. 10, pp. 2146-2157, 2001.

[21] J. Tekstra, C. E. Visser, C. W. Tuk et al., "Identification of the major chemokines that regulate cell influxes in peritoneal dialysis patients," Journal of the American Society of Nephrology, vol. 7, no. 11, pp. 2379-2384, 1996.

[22] C. Verger, A. Luger, H. L. Moore, and K. D. Nolph, "Acute changes in peritoneal morphology and transport properties with infectious peritonitis and mechanical injury," Kidney International, vol. 23, no. 6, pp. 823-831, 1983.

[23] Y. L. Kim, S. H. Kim, J. H. Kim et al., "Effects of peritoneal rest on peritoneal transport and peritoneal membrane thickening in continuous ambulatory peritoneal dialysis rats," Peritoneal Dialysis International, vol. 19, supplement 2, pp. S384-S387, 1999.

[24] M. Zareie, E. D. Keuning, P. M. ter Wee, R. H. J. Beelen, and J. van den Born, "Peritoneal dialysis fluid-induced changes of the peritoneal membrane are reversible after peritoneal rest in rats," Nephrology Dialysis Transplantation, vol. 20, no. 1, pp. 189-193, 2005.

[25] P. Fabbrini, M. Zareie, P. M. ter Wee, E. D. Keuning, R. H. J. Beelen, and J. van den Born, "Peritoneal exposure model in the rat as a tool to unravel bio(in)compatibility of PDF," Nephrology Dialysis Transplantation, vol. 21, supplement 2, pp. ii8-ii11, 2006.

[26] P. Margetts, "Heparin and the peritoneal membrane," Peritoneal Dialysis International, vol. 29, no. 1, pp. 16-19, 2009.

[27] A. W. D. Stavenuiter, M. N. Schilte, P. M. ter Wee, and R. H. J. Beelen, "Angiogenesis in peritoneal dialysis," Kidney and Blood Pressure Research, vol. 34, no. 4, pp. 245-252, 2011.

[28] L. S. Aroeira, E. Lara-Pezzi, J. Loureiro et al., "Cyclooxygenase2 mediates dialysate-induced alterations of the peritoneal membrane," Journal of the American Society of Nephrology, vol. 20, pp. 582-592, 2009.

[29] P. Fabbrini, M. N. Schilte, M. Zareie et al., "Celecoxib treatment reduces peritoneal fibrosis and angiogenesis and prevents ultrafiltration failure in experimental peritoneal dialysis," Nephrology Dialysis Transplantation, vol. 24, no. 12, pp. 3669-3676, 2009.

[30] T. Bellón, V. Martínez, B. Lucendo et al., "Alternative activation of macrophages in human peritoneum: implications for peritoneal fibrosis ," Nephrology Dialysis Transplantation. In press.

[31] B. M. Delavary, W. M. van der Veer, M. van Egmond, F. B. Niessen, and R. H. J. Beelen, "Macrophages in skin injury and repair," Immunobiology, vol. 216, no. 7, pp. 753-762, 2011.

[32] G. J. van Der Bij, S. J. Oosterling, S. Meijer, R. H. J. Beelen, and M. van Egmond, "The role of macrophages in tumor development," Cellular Oncology, vol. 27, no. 4, pp. 203-213, 2005.

[33] S. J. Oosterling, G. van der Bij, G. A. Meijer, S. Meijer, R. H. J. Beelen, and M. van Egmond, "Macrophages direct microscopic phenotype and clinical outcome in a colon cancer 
model," European Journal of Cancer Supplements, vol. 3, p. 194, 2005.

[34] R. Kalluri and E. G. Neilson, "Epithelial-mesenchymal transition and its implications for fibrosis," Journal of Clinical Investigation, vol. 112, no. 12, pp. 1776-1784, 2003.

[35] D. Brancaccio, M. Cozzolino, S. Pasho, G. Fallabrino, L. Olivi, and M. Gallieni, "New acquisitions in therapy of secondary hyperparathyroidism in chronic kidney disease and peritoneal dialysis patients: role of vitamin D receptor activators," Peritoneal Dialysis, vol. 163, pp. 219-226, 2009.

[36] J. Loureiro, M. Schilte, A. Aguilera et al., "BMP-7 blocks mesenchymal conversion of mesothelial cells and prevents peritoneal damage induced by dialysis fluid exposure," Nephrology Dialysis Transplantation, vol. 25, no. 4, pp. 1098-1108, 2010.

[37] M. N. Schilte, E. D. Keuning, and P. M. ter Wee, "Vitamin D receptor activation infuences peritoneal function and remodelling in experimental peritoneal dialysis," in Proceedings of the EuroPD Meeting, 2009. 


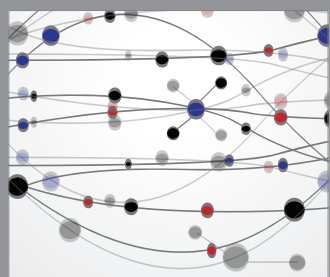

The Scientific World Journal
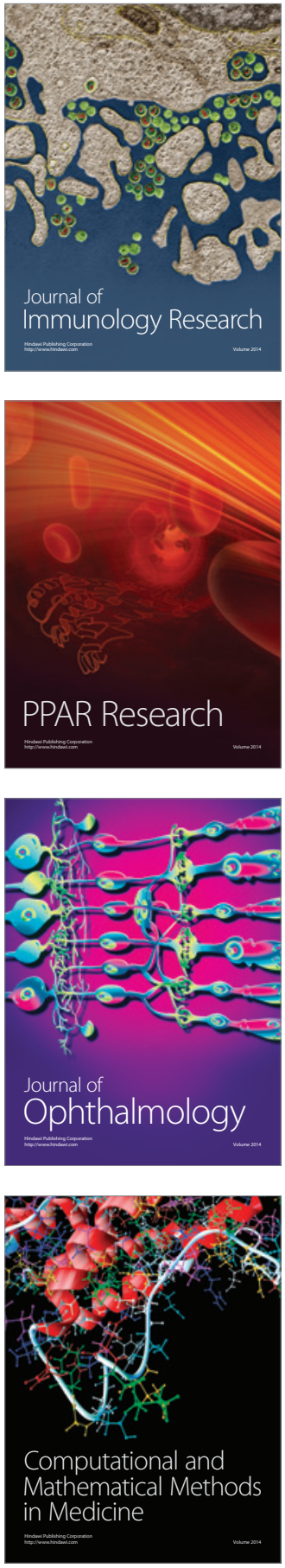

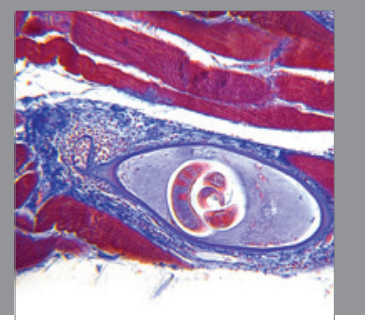

Gastroenterology

Research and Practice
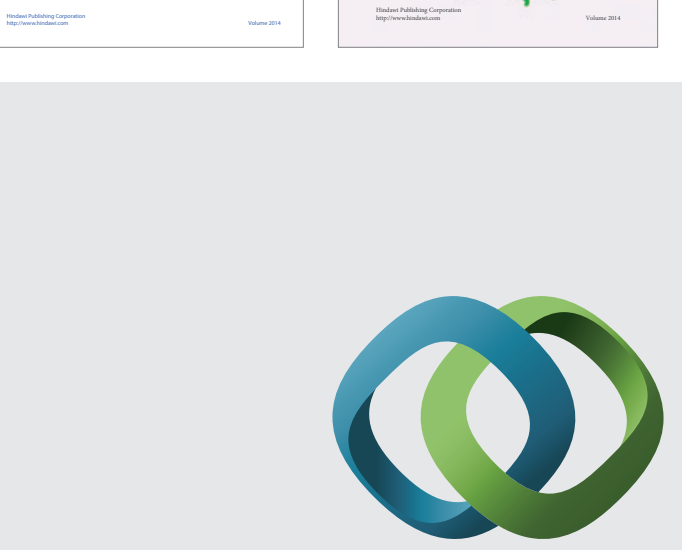

\section{Hindawi}

Submit your manuscripts at

http://www.hindawi.com
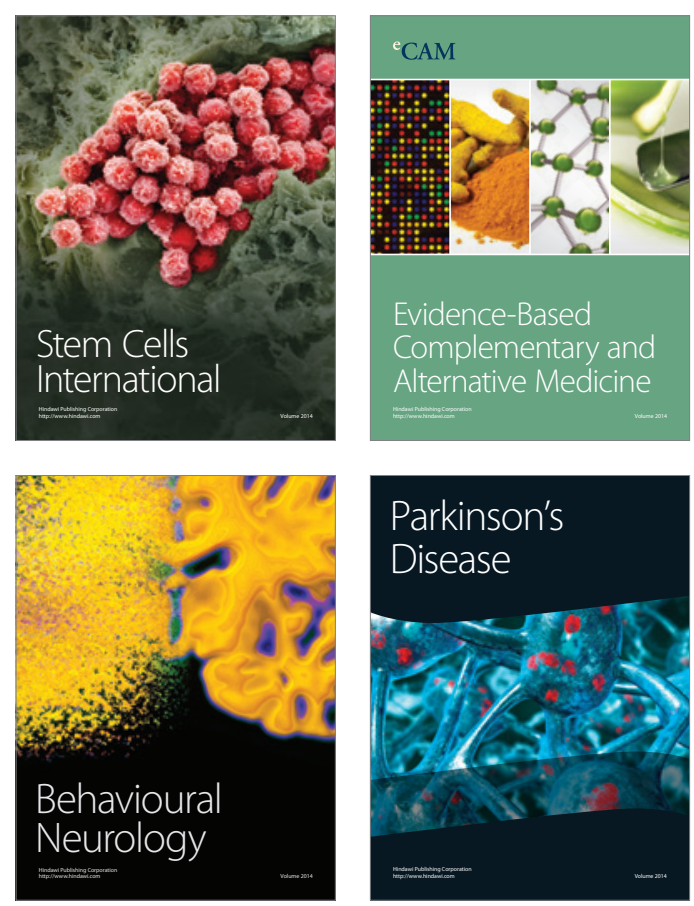

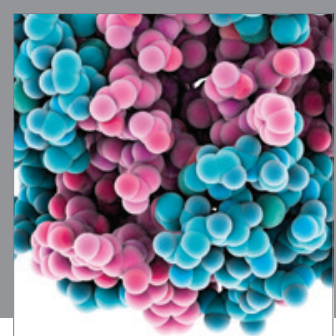

Journal of
Diabetes Research

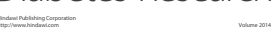

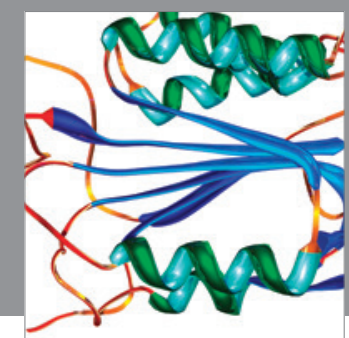

Disease Markers
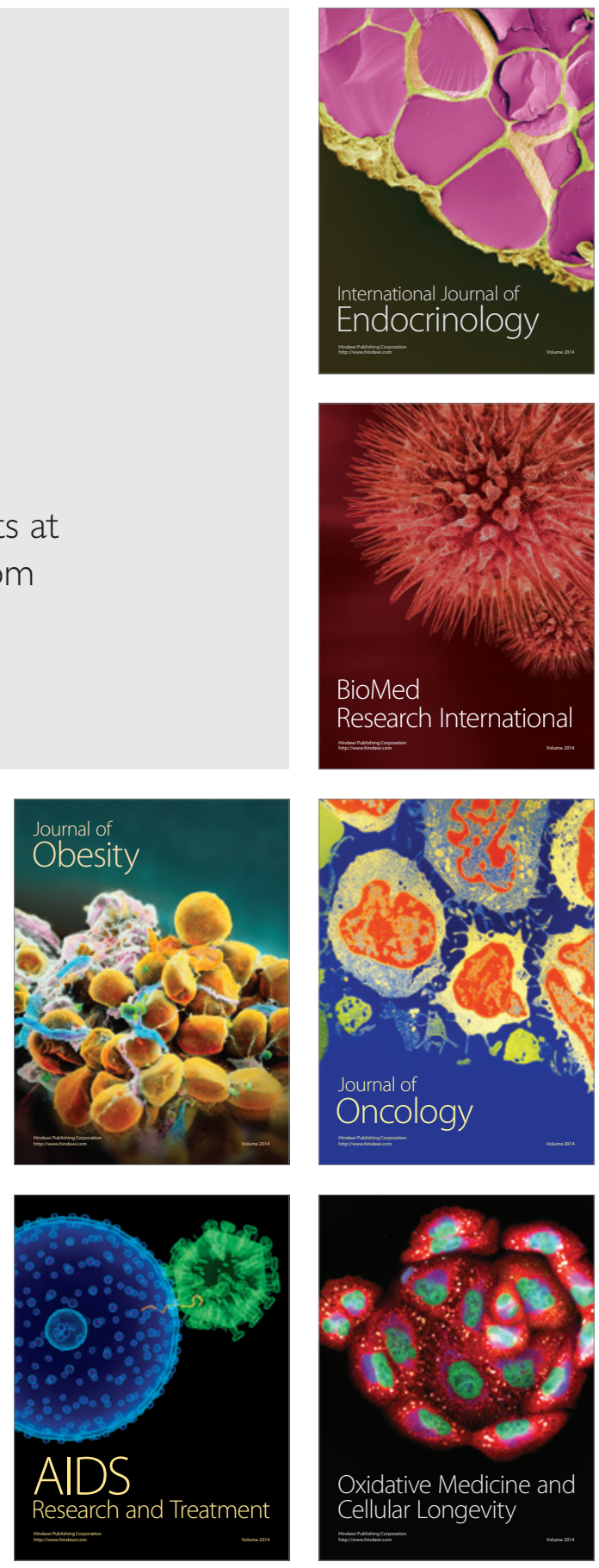